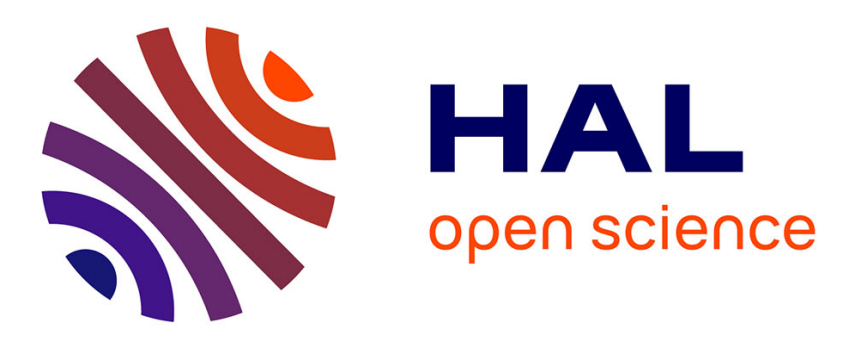

\title{
Robust Design of a Switched PI Controller for an Uncertain Traffic Model
}

Antoine Lemarchand, Damien Koenig, John Jairo Martinez Molina

\section{To cite this version:}

Antoine Lemarchand, Damien Koenig, John Jairo Martinez Molina. Robust Design of a Switched PI Controller for an Uncertain Traffic Model. CDC 2010 - 49th IEEE Conference on Decision and Control, Dec 2010, Atlanta, Georgie, United States. pp.n-c. hal-00544103

\section{HAL Id: hal-00544103 https://hal.science/hal-00544103}

Submitted on 7 Dec 2010

HAL is a multi-disciplinary open access archive for the deposit and dissemination of scientific research documents, whether they are published or not. The documents may come from teaching and research institutions in France or abroad, or from public or private research centers.
L'archive ouverte pluridisciplinaire HAL, est destinée au dépôt et à la diffusion de documents scientifiques de niveau recherche, publiés ou non, émanant des établissements d'enseignement et de recherche français ou étrangers, des laboratoires publics ou privés. 


\title{
Robust Design of a Switched PI Controller for an Uncertain Traffic Model
}

\author{
Antoine Lemarchand, Damien Koenig and John J. Martinez
}

\begin{abstract}
This paper deals with the robust local $H_{\infty}$ regulation of a freeway section. The model used to describe the onramp neighborhood is a discrete-time linear switching model. We extended this model with parametric uncertainties. We propose to design a bank of robust PI controllers to ensure that the system follows the concentration references even in presence of parametric uncertainties and exogenous disturbances. These controllers are computed using LMI resolution.
\end{abstract}

\section{INTRODUCTION}

The amount of vehicles on roads increases every day, and causes a waste of time and money [13]. With the construction of new roads, traffic control via on-ramp metering is one of the most explored ways to deal with this problem. The onramp metering aims at the improving of traffic condition by controlling the inflow of freeways.

Most of the solution proposed are based on a hierarchical architecture [14],[7],[9]. This architecture is constituted by the high level ( $\Leftrightarrow$ optimization layer) and by the low level ( $\Leftrightarrow$ local controller level).

The high level layer computes the optimal references (concentrations) to be tracked by the system. This layer is usually based on linear programming algorithms [6],[7],[4]. These references are open loop calculation based on a nominal model. Due to parametric uncertainties and disturbances, the optimal solution computed by this algorithm can't be applied directly on the system.

Therefore, we use the low level layer to ensure that the system really tracks optimal references. In this layer, references are locally applied to on-ramps neighborhoods as shown in Figure 1. Several approaches have been explored to solve this issue such as ALINEA [5], and others such as [15],[16]. In this article, we focus on the local regulation layer. We assume that the optimal trajectories computed by the optimization layer are known. Since the parameters of the model are strongly uncertain, the Cell Transmission Model (CTM) is extended with a model of uncertainties. Thanks to this model, we design a bank of robust switched PI controller which guarantee a certain attenuation of the $H_{\infty}$ norm of disturbance transfer function.

The paper is organised as follows. A brief presentation of the CTM is provided in Section II. This model is extended with parametric uncertainties and written as a discrete-time switching uncertain model (Section III). The design of robust switched PI controller via LMI formulation is presented in Section IV. Some simulation results are provided in Section V. In Section VI, some concluding remarks end the paper.

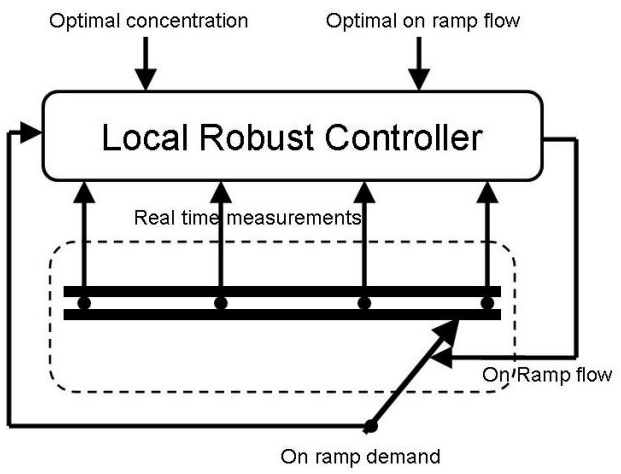

Fig. 1. Local regulation.

\section{Cell Transmission Model}

The CTM is a first order linear discrete traffic model proposed in [2],[1] and used in many traffic issues. The CTM model is presented in three parts: Section II-A presents the elementary cells and Section II-B presents the junction model.

\section{A. Elementary Cell}

Consider the road section divided into elementary cells. Assuming that in each cell, the density, $\rho(k)$, of vehicles is homogenous. Then $\rho(k)$ can be related to $\phi_{i}(k)$ and $\phi_{i+1}(k)$ (respectively the flow entering and leaving cell $i$ ) by

$$
\rho_{i}(k+1)=\rho_{i}(k)+\frac{T}{l_{i}}\left(\phi_{i}(k)-\phi_{i+1}(k)\right),
$$

as depicted in Figure 2

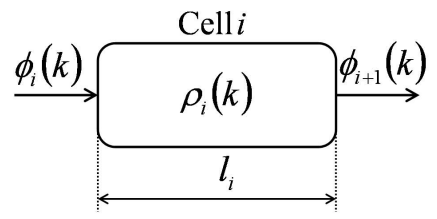

Fig. 2. Elementary cell.

where $l_{i}$ is the length of cell $i$ and $T$ the period of discretized time. To guarantee numerical stability, $T$ has to fit the condition $T<\inf \left(\frac{l_{i}}{v_{i}}\right), \forall i=1, \ldots, N$ ( $N$, the number of cells). Notice that (1) is the conservation law of vehicles [8]. 


\section{B. Junctions}

Assuming that a cell can contain at most one on ramp at the beginning of the cell and one off ramp at the end as depicted in Figure 3. Denote $u_{i}$ the flow entering cell $i$ via on ramp, and $\phi_{\text {out }_{i}}$ the flow leaving cell $i-1$ via off ramp. We consider that off-ramps have infinite capacities (i.e. no congestion propagates in the freeway section via off-ramps)

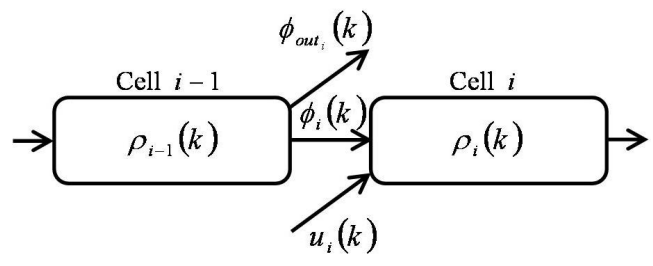

Fig. 3. Junction.

Define $S_{\phi_{i}}(k)$ as the flow that upstream cell can supply, and $R_{\phi_{i}}(k)$ as the flow that downstream cell can receive. $S_{\phi_{i}}(k)$ and $R_{\phi_{i}}(k)$ are characterized by

$$
\begin{aligned}
S_{\phi_{i}}(k) & =\min \left(\left(1-\beta_{i}\right) \cdot v_{i-1} \cdot \rho_{i-1}(k), \phi_{M_{i}}\right), \\
R_{\phi_{i}}(k) & =\min \left(w_{i} \cdot\left(\rho_{J_{i}}-\rho_{i}(k)\right)-u_{i}(k), \phi_{M_{i}}\right) .
\end{aligned}
$$

with $v_{i},(\mathrm{~km} / \mathrm{h})$ the free flow speed, $w_{i}$ the backward congestion propagation speed, $\rho_{J_{i}},(v e h / \mathrm{km})$ the jam density (i.e. maximal density), and $\phi_{M_{i}},(v e h / h)$ the maximum flow that can travel from upstream to downstream cell and $\beta_{i}$ the split ratio (i.e. the part of the flow going out of cell $i-1$ that leave main road via off ramp) defined by

$$
\beta_{i}=\frac{\phi_{\text {out }_{i}}(k)}{\phi_{\text {out }_{i}}(k)+\phi_{i}(k)} .
$$

Obviously the flow moving from upstream to downstream cell is the minimum of (2) and (3), i.e.

$$
\phi_{i}(k)=\min \left(S_{\phi_{i}}(k), R_{\phi_{i}}(k)\right) .
$$

\section{Junction modes:}

From (2), (3), and (5) one can identify the three possible modes of the junction: A free mode $(F)$ where the flow is proportional to the upstream cell concentration, a decoupled mode (D) where the flow is equal to the maximal flow, and a congested mode (C) where the flow is proportional to the remaining space in downstream cell. A graphical representation of (2), (3) and (5) is provided in Figure 4. It is called the fundamental diagram. This kind of diagram appears in every traffic issues using macroscopic model [3].

\section{UNCERTAIN SWITCHED CTM}

\section{A. Uncertain Parameters}

The nominal parameters of the fundamental diagram can be computed using the calibration methods described in [12] with experimental datas ${ }^{1}$. The obtain diagram contains

\footnotetext{
${ }^{1}$ Real time measurements realized on D383 road (near Lyon, France) provided by DDE69
}

strong uncertainties. They can be modeled as the following parametric uncertainties:

$$
\begin{aligned}
v_{i-1}(k) & =v_{0_{i-1}}+\Delta v_{i-1}(k), \\
\phi_{M_{i}}(k) & =\phi_{M_{0_{i}}}+\Delta \phi_{M_{i}}(k), \\
w_{i}(k) & =w_{0_{i}}+\Delta w_{i}(k),
\end{aligned}
$$

where $v_{0}, \phi_{M_{0}}$ and $w_{0}$ are respectively the nominal free flow speed, maximum flow, and backward congestion propagation speed computed thanks to [12], and $\Delta v_{0}, \Delta \phi_{M_{0}}$ and $\Delta w_{0}$ the corresponding uncertainties. The new uncertain fundamental diagram is depicted in Figure 4.

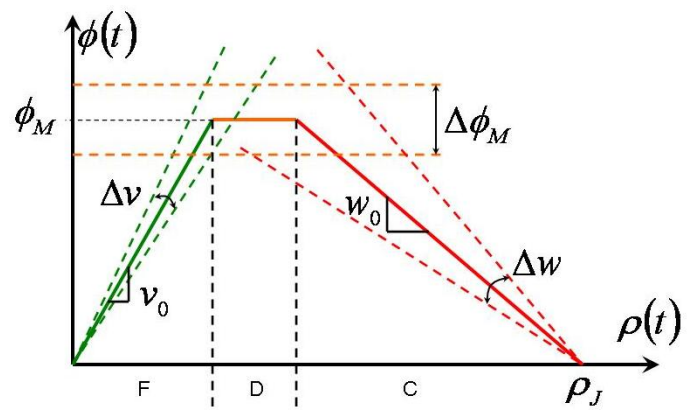

Fig. 4. Fundamental diagram with parametric uncertainties.

\section{B. Matrix formulation}

Consider a freeway section, divided into $N$ cells, $N+1$ junctions and $N_{i n}$ inputs. Define

$$
\alpha(k):=\left[\alpha_{1}(k), \cdots, \alpha_{N+1}(k)\right]
$$

where $\alpha_{i}(k) \in\{F, D, C\}$ represents the mode of junction $i$ presented in Section II-B.

Proposition 3.1: For all $\alpha(k)$ the dynamics of a freeway section can be written as an uncertain dynamical system:

$$
\begin{aligned}
\rho(k+1)= & \left(A_{0_{\alpha(k)}}+F_{\alpha(k)} \Delta(k) G\right) \cdot \rho(k) \\
& +B_{\alpha(k)} \cdot u(k)+E_{\alpha(k)} \cdot d(k)
\end{aligned}
$$

where $\rho(k)=\left[\rho_{1}(k), \ldots, \rho_{N}(k)\right]$ is the state vector of vehicle densities for each cell, $u(k)$ the on ramp controlled flows and $d(k)$ a vector of external disturbances. $A_{0_{\alpha(k)}}, B_{\alpha(k)}, E_{\alpha(k)}, F_{\alpha(k)}, G$ are know matrices of appropriate dimensions. Each matrices and vectors are defined bellow. (see (11-14) and (16-17))

Proof: First, the conservation law (1) is rewritten as follows:

$$
\rho(k+1)=\rho(k)+\Theta \cdot \phi(k)+B \cdot u(k)
$$

where $\phi=\left[\phi_{1}, \cdots, \phi_{N+1}\right]$ is the vector of flows in each junctions, and

$$
\Theta=\left(\begin{array}{cccc}
\frac{T}{l_{1}} & -\frac{T}{l_{1}} & 0 & 0 \\
0 & \ddots & \ddots & 0 \\
0 & 0 & \frac{T}{l_{N}} & -\frac{T}{l_{N}}
\end{array}\right),
$$




$$
B=\left(\begin{array}{ccc}
0 & 0 & 0 \\
\frac{T}{l_{i 1}} & 0 & \vdots \\
0 & \frac{T}{l_{i x}} & \vdots \\
\vdots & 0 & 0 \\
0 & 0 & \frac{T}{l_{i N_{i n}}}
\end{array}\right) .
$$

Secondly, the vector $\phi(k)$ is reformulated thanks to the results of Section II-B with a matrix formulation as follows:

$$
\begin{gathered}
\phi(k)=A_{\phi_{\alpha(k)}}(k) \cdot \rho(k)+B_{\phi_{\alpha(k)}} \cdot u(k)+E_{\phi_{\alpha(k)}} \cdot d(k) \\
+a_{J_{\alpha(k)}}(k) \cdot \rho_{J}+a_{\phi_{M \alpha(k)}} \cdot \phi_{M}(k),
\end{gathered}
$$

where, $\rho_{J}:=\left[\rho_{J_{1}}, \cdots, \rho_{J_{N+1}}\right]$ is the vector jam densities of each cell, $\phi_{M}(k):=\left[\phi_{M_{1}}(k), \cdots, \phi_{M_{N+1}}(k)\right]$ is the vector of maximal flows in each junction (see Figure 4$), d(k)$ the boundary conditions, and the matrices $A_{\phi_{\alpha(k)}}(k), B_{\phi_{\alpha(k)}}, E_{\phi_{\alpha(k)}}, a_{J_{\alpha(k)}}(k), a_{\phi_{M \alpha(k)}}$ are respectively described by:

$$
\begin{aligned}
& A_{\phi_{\alpha(k)}}(k)=\left(\begin{array}{ccc}
-w_{1}(k) \cdot e_{C_{1}} & 0 & 0 \\
v_{1}(k) \cdot e_{F_{2}} & \ddots & 0 \\
0 & \ddots & -w_{N}(k) \cdot e_{C_{N}} \\
0 & 0 & v_{N}(k) \cdot e_{F_{N+1}}
\end{array}\right), \\
& B_{\phi_{\alpha(k)}}=\left(\begin{array}{ccc}
0 & 0 & 0 \\
-e_{C_{1}} & 0 & \vdots \\
0 & -e_{C_{x}} & \vdots \\
\vdots & 0 & 0 \\
0 & 0 & -e_{C_{N i n}}
\end{array}\right) \\
& E_{\phi_{\alpha(k)}}=\left(\begin{array}{cc}
e_{F_{1}} & 0 \\
0 & \vdots \\
\vdots & 0 \\
0 & e_{C_{N+1}}
\end{array}\right) \\
& a_{J_{\alpha(k)}}(k)=\left(\begin{array}{ccc}
e_{C_{1}} w_{1}(k) & & 0 \\
& \ddots & \\
0 & & e_{C_{N+1}} w_{N+1}(k)
\end{array}\right), \\
& a_{\phi_{M \alpha(k)}}=\left(\begin{array}{ccc}
e_{D_{1}} & & 0 \\
& \ddots & \\
0 & & e_{D_{N}}
\end{array}\right),
\end{aligned}
$$

with,

$$
\begin{aligned}
e_{F_{i}} & = \begin{cases}1 & \text { if junction } i \text { is Free } \\
0 & \text { otherwise }\end{cases} \\
e_{D_{i}} & = \begin{cases}1 & \text { if junction } i \text { is Decoupled } \\
0 & \text { otherwise }\end{cases} \\
e_{C_{i}} & = \begin{cases}1 & \text { if junction } i \text { is Congested } \\
0 & \text { otherwise }\end{cases}
\end{aligned}
$$

Substituting (10) into (9) and using the relations (11-14) the formulation (15) is obtained.

$$
\begin{aligned}
A_{\alpha(k)}(k)= & I+\Theta \cdot A_{\phi_{\alpha(k)}}(k) \\
B_{\alpha(k)}= & B+\Theta \cdot B_{\phi_{\alpha(k)}} \\
E_{d_{\alpha(k)}=}= & \Theta \cdot E_{\phi_{\alpha(k)}} \\
a_{\alpha(k)}(k)= & \Theta \cdot\left(a_{J_{\alpha(k)}}(k) \cdot \rho_{J}+a_{\phi_{M \alpha(k)}} \cdot \phi_{M}(k)\right) \cdot(1) \\
\rho(k+1)= & A_{\alpha(k)}(k) \cdot \rho(k)+B_{\alpha(k)} \cdot u(k) \\
& +E_{d_{\alpha(k)}} \cdot d(k)+a_{\alpha(k)}(k) .
\end{aligned}
$$

Notice that (11) and (14) contains uncertain parameters. Let's separate the nominal parts and the uncertain parts of (11) and (14). From (6), the term (11) can be decomposed as a nominal and uncertain part:

$$
A_{\alpha(k)}(k)=A_{0_{\alpha(k)}}+F_{\alpha(k)} \cdot \Delta(k) \cdot G
$$

with,

$$
\begin{gathered}
A_{0_{\alpha(k)}}=\left.A_{\alpha(k)}(k)\right|_{v_{i}(k)=v_{0_{i}}, w_{i}(k)=w_{0_{i}}}, \\
F_{\alpha(k)}=\Theta \cdot\left(\begin{array}{cccccc}
0 & \cdots & 0 & e_{C_{1}} \cdot & & 0 \\
e_{F_{2}} & & 0 & & \ddots & \\
& \ddots & & 0 & & e_{C_{N}} \\
0 & & e_{F_{N+1}} & 0 & \ldots & 0
\end{array}\right), \\
G=\left(\begin{array}{cccc}
\operatorname{diag}\left(\left\|\Delta v_{i}\right\|_{\infty}\right)_{2}^{N+1} & & 0 & \\
0 & & \operatorname{diag}\left(\left\|\Delta w_{i}\right\|_{\infty}\right)_{1}^{N}
\end{array}\right)
\end{gathered}
$$

and,

$$
\begin{aligned}
& \Delta(k)=\left(\begin{array}{cc}
\operatorname{diag}\left(\delta v_{i}(k)\right)_{2}^{N+1} & 0 \\
0 & \operatorname{diag}\left(\delta w_{i}(k)\right)_{1}^{N}
\end{array}\right) \\
& \delta v_{i}(k)=\frac{\Delta v_{i}(k)}{\left\|\Delta v_{i}\right\|_{\infty}} \\
& \delta w_{i}(k)=\frac{\Delta w_{i}(k)}{\left\|\Delta w_{i}\right\|_{\infty}} \\
& \text { so } \Delta(k)^{T} \Delta(k)<1 .
\end{aligned}
$$

From (6) the term (14) can be written as an external disturbance:

$a_{\alpha(k)}(k)=E_{\phi_{M_{\alpha(k)}}} . \phi_{M}(k)+E_{J_{\alpha(k)}} . \rho_{J}+E_{w_{\alpha(k)}} . w_{\Delta}(k)$

with:

$$
\begin{aligned}
E_{\phi_{M_{\alpha}(k)}} & =\Theta \cdot a_{\phi_{M \alpha(k)}} \\
E_{J_{\alpha(k)}} & =\left.\Theta \cdot a_{J_{\alpha(k)}}(k)\right|_{w_{i}(k)=w_{0_{i}}} \\
E_{w_{\alpha(k)}} & =\Theta \cdot \operatorname{diag}\left(e_{C_{i}}\right)_{1}^{N+1} \\
E_{\Delta}(k) & =\left(\begin{array}{c}
\Delta w_{1}(k) \cdot \rho_{J_{1}} \\
\vdots \\
\Delta w_{N+1}(k) \cdot \rho_{J_{N+1}}
\end{array}\right)
\end{aligned}
$$

Substituting (16) and (17) in (15), the formulation (8) of the proposition is obtained. 


\section{REGULATOR DESIGN}

\section{A. Study case}

For our study, we consider that optimal trajectories $\left(\rho^{*}(k)\right.$ for mainline concentration and $u^{*}(k)$ for on-ramp flow are known. Without loss of generality, we consider the neighborhood depicted in Figure 5.

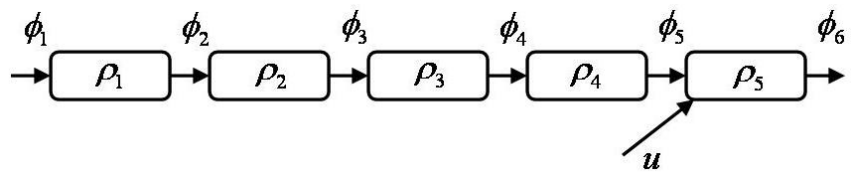

Fig. 5. On-ramp neighborhood.

We consider here that in normal traffic condition (i.e. without accidents) the different mode of the section that can occurs are the following:

- case $1: \alpha(k)=[F F F F F F]$

- case $2: \alpha(k)=[F F F F F D]$

- case $3: \alpha(k)=[F F F F C D]$

- case $4: \alpha(k)=[F F F C C D]$

- case $5: \alpha(k)=[F F C C C D]$

- case $6: \alpha(k)=[F C C C C D]$

$\alpha(k)$ defined in 7 . We notate $\Omega$ this set of case. In case 1 , all the junctions of the section are in free mode $(F)$. Just before congestion appears (case 2) junction 4 switches to decoupled mode (D). Then in case $3,4,5$ and 6 , the congestion propagates backward (i.e. junction 5 to 2 switch to congested mode $(\mathrm{C})$ ). We denote $\tau_{i j}$ the transition from case $i$ to case $j$. So the set of possible transition is:

$$
\Upsilon=\left\{\tau_{11}, \tau_{12}, \tau_{21}, \tau_{22}, \cdots, \tau_{55}, \tau_{65}, \tau_{66}\right\}
$$

\section{B. Extended system}

The problem now, is to design a switched PI controller in order to stabilize the system and attenuate the disturbances. For this purpose, we have to extend our system with an integrator. To keep the stabilizability property of the system, we can only extend one state of the system with an integrator. For the case 1 and 2, we choose to extend the system with an integrator on $\epsilon_{5}(k)$ (where $\epsilon_{5}(k)=\rho_{5}(k)-\rho_{5}^{*}(k)$ ). For the case 3 to 6 , we choose to extend the system with an integrator on the density of the cell where the congestion front stand (respectively $\epsilon_{4}(k)$ to $\epsilon_{1}(k)$ ). So, the new state vector becomes

$$
X(k)=\left(\begin{array}{l}
\rho(k) \\
z(k)
\end{array}\right)
$$

with:

$$
z(k+1)=z(k)+ \begin{cases}\epsilon_{5}(k) & , \text { if case } 1 \text { or } 2 \\ \epsilon_{4}(k) & , \text { if case } 3 \\ \epsilon_{3}(k) & , \text { if case } 4 \\ \epsilon_{2}(k) & \text {, if case } 5 \\ \epsilon_{1}(k) & , \text { if case } 6\end{cases}
$$

The extended system is described in a compact form as follows:

$$
\left\{\begin{aligned}
X(k+1)= & \left(A_{a_{\alpha(k)}}+F_{a_{\alpha(k)}} \cdot \Delta(k) \cdot G_{a}\right) \cdot X(k) \\
& +B_{a_{\alpha(k)}} \cdot u(k)+E_{a_{\alpha(k)}} \cdot w(k) \\
Z(k)= & C_{a_{\alpha(k)}} \cdot X(k)
\end{aligned}\right.
$$

with

$$
\begin{aligned}
& A_{a_{\alpha(k)}}=\left(\begin{array}{ll}
A_{0_{\alpha(k)}} & 0 \\
A_{z_{\alpha(k)}} & 1
\end{array}\right), \\
& F_{a_{\alpha(k)}}=\left(\begin{array}{c}
F_{1_{\alpha(k)}} \\
0
\end{array}\right) \text {, } \\
& G_{a}=\left(\begin{array}{ll}
G & 0
\end{array}\right) \text {, } \\
& B_{a_{\alpha(k)}}=\left(\begin{array}{c}
B_{\alpha(k)} \\
0
\end{array}\right) \text {, } \\
& E_{a_{\alpha(k)}}=\left(\begin{array}{ccc}
f_{d} \cdot E_{\alpha(k)} & 0 & f_{\phi} \cdot H_{\phi_{M_{\alpha}(k)}} \\
0 & -f_{z} \cdot A_{z_{\alpha(k)}} & 0 \\
f_{J} \cdot H_{J_{\alpha(k)}} & f_{w} \cdot H_{w_{\alpha(k)}}
\end{array}\right),{ } \\
& \left.\begin{array}{cc}
0 & w \cdot H_{\alpha(k)}
\end{array}\right), \\
& C_{a_{\alpha(k)}}=\left(\begin{array}{ll}
A_{z_{\alpha(k)}} & 0
\end{array}\right), \\
& \bar{d}(k)=\left(\begin{array}{lllll}
d(k) & \rho^{*}(k) & \phi_{M}(k) & \rho_{J} & w_{\Delta}(k)
\end{array}\right)^{T},
\end{aligned}
$$

According to (24), we have

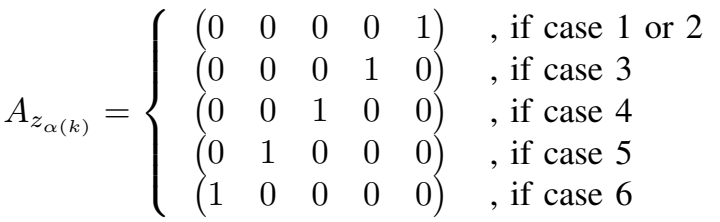

$f_{d}, f_{z}, f_{\phi}, f_{J}$ and $f_{w}$ are added to the matrix $E_{a_{\alpha(k)}}$ to weight the influence of the different perturbations for the $H_{\infty}$ attenuation criteria.

\section{LMI formulation}

Take the following notations, $\alpha(k)=i, \alpha(k+1)=j$. Assume that each cell density is measured. This seems to be a fair assumption since sensor becomes cheaper and cheaper, and since much roads are equipped of sensors.

Proposition 4.1: if $\exists Q_{i}=Q_{i}^{T}>0, e_{i}>0, U_{i}, i \in \Omega$ such that (26) holds for all transition $\tau_{i j} \in \Upsilon$,

Then:

- The extended system (25) is stable under state feedback $u(k)=-K_{i} X(k), K_{i}=U_{i} Q_{i}^{-1}$,

- The poles of the system are placed in a circle of center $\sigma$ and radius $r$,

- The $H_{\infty}$ norm of the transfert function between $w(k)$ and $Z(k)$ is bounded by $\gamma$ (weighted by $f_{d}, f_{z}, f_{\phi}, f_{J}$ and $\left.f_{w}\right)$.

Proof: The core of our approach is the use of the following candidate Lyapunov function

$$
V_{i}(k)=X(k)^{T} P_{i} X(k) .
$$

Where $P_{i}$ is a positive definite matrix. Since $P_{i}=P_{i}^{T}>0$. We can write the following $H_{\infty}$ attenuation criteria:

$$
\begin{gathered}
X(k+1)^{T} P_{j} X(k+1)-X(k)^{T} P_{i} X(k) \\
\quad+Z(k)^{T} Z(k)-\gamma^{2} w(k)^{T} w(k)<0
\end{gathered}
$$




$$
\left(\begin{array}{ccccc}
\epsilon_{i} F_{a_{i}} F_{a_{i}}^{T}-r Q_{j} & A_{a_{i}} Q_{i}+B_{a_{i}} U_{i}-\sigma Q_{i} & r E_{a_{i}} & 0 & 0 \\
* & -r Q_{i} & 0 & Q_{i} G_{a}^{T} & Q_{i} C_{a}^{T} \\
* & * & -r \gamma^{2} I & 0 & 0 \\
* & * & * & -\epsilon_{i} I & 0 \\
* & * & * & * & -r^{-1} I
\end{array}\right)<0
$$

Substituting (25) in (28), the following condition is obtained:

$$
\begin{gathered}
\left(\left(A_{a_{i}}+F_{a_{i}} \cdot \Delta(k) \cdot G_{a}-B_{a_{\alpha(k)}} K_{i}\right) \cdot X(k)+E_{a_{i}} \cdot \bar{d}(k)\right)^{T} \\
\cdot P_{j}\left(\left(A_{a_{i}}+F_{a_{i}} \cdot \Delta(k) \cdot G_{a}-B_{a_{\alpha(k)}} K_{i}\right) \cdot X(k)\right. \\
\left.+E_{a_{i}} \cdot \bar{d}(k)\right)-X(k)^{T} P_{i} X(k)+\left(C_{i} X(k)\right)^{T}\left(C_{i} X(k)\right) \\
-\gamma^{2} \bar{d}(k)^{T} \bar{d}(k)<0
\end{gathered}
$$

Taking the notation $A_{a_{i}}-B_{a_{i}} K_{i}=\Gamma_{i}$ the previous inequality is equivalent to:

$$
\begin{array}{cc}
\Pi_{11}^{1}= & \left(\begin{array}{cc}
\Pi_{11}^{1} & \Pi_{21}^{1} \\
* & E_{a_{i}}^{T} E_{a_{i}}-\gamma^{2} I
\end{array}\right)<0 \\
\Pi_{21}^{1}= & \left.F_{a_{i}} \cdot \Delta(k) \cdot G_{a}\right)^{T} P_{j}\left(\Gamma_{i}+F_{a_{i}} \cdot \Delta(k) \cdot G_{a}\right) \\
-P_{i}+C_{i}^{T} C_{i} \\
\left(\Gamma_{i}+F_{a_{i}} \cdot \Delta(k) \cdot G_{a}\right)^{T} P_{j} E_{a_{i}}
\end{array}
$$

Applying Schur complement, we obtain the following inequality:

$$
\left(\begin{array}{ccc}
-P_{i}+C_{i}^{T} C_{i} & 0 & \left(\Gamma_{i}+F_{a_{i}} \cdot \Delta(k) \cdot G_{a}\right)^{T} \\
* & -\gamma^{2} I & E_{a_{i}} \\
* & * & -P_{j}^{-1}
\end{array}\right)<0
$$

Using majoration lemma, we can demonstrate that (31) holds if $\exists \epsilon_{i}>0$ such that

$$
\left(\begin{array}{ccc}
-P_{i}+C_{i}^{T} C_{i} & 0 & \Gamma_{i}^{T} \\
+\epsilon_{i}^{-1} G_{a}^{T} G_{a} & 0 & E_{a_{i}} \\
* & -\gamma^{2} I & -P_{j}^{-1}+\epsilon_{i} F_{a_{i}} F_{a_{i}}^{T}
\end{array}\right)<0
$$

Applying the Schur complement

$$
\begin{gathered}
\left(\begin{array}{cc}
-P_{i}+C_{i}^{T} C_{i}+\epsilon_{i}^{-1} G_{a}^{T} G_{a} & 0 \\
* & -\gamma^{2} I
\end{array}\right) \\
-\left(\begin{array}{c}
\Gamma_{i}^{T} \\
E_{a_{i}}^{T}
\end{array}\right)\left(P_{j}^{-1}-\epsilon_{i} F_{a_{i}} F_{a_{i}}^{T}\right)^{-1}\left(\begin{array}{cc}
\Gamma_{i} & E_{a_{i}}
\end{array}\right)<0
\end{gathered}
$$

Multiplying both side of (33) by $\left(\begin{array}{cc}P i^{-1} & 0 \\ 0 & I\end{array}\right)$ and applying Schur complement, (34) is obtain.

$$
\begin{gathered}
\left(\begin{array}{ccc}
-P_{j}^{-1}+\epsilon_{i} F_{a_{i}} F_{a_{i}}^{T} & \Gamma P_{i}^{-1} & E_{a_{i}} \\
* & \Pi_{22}^{2} & 0 \\
* & * & -\gamma^{2} I
\end{array}\right)<0 \\
\Pi_{22}^{2}=-P_{i}^{-1}+P_{i}^{-1} C_{i}^{T} C_{i} P_{i}^{-1}+\epsilon_{i}^{-1} P_{i}^{-1} G_{a}^{T} G_{a} P_{i}^{-1}
\end{gathered}
$$

Applying twice Schur lemma on term $\Pi_{22}^{2}$, (34) becomes:

$$
\left(\begin{array}{ccccc}
-P_{j}^{-1}+\epsilon_{i} F_{a_{i}} F_{a_{i}}^{T} & \Gamma P_{i}^{-1} & E_{a_{i}} & 0 & 0 \\
* & -P_{i}^{-1} & 0 & P_{i}^{-1} G_{a}^{T} & P_{i}^{-1} C_{i}^{T} \\
* & * & -\gamma^{2} I & 0 & 0 \\
* & * & * & -\epsilon_{i} I & 0 \\
* & * & * & * & -I
\end{array}\right)
$$

It is stated in that In order to improve the performance, the poles of the closed loop system are placed in a particular region. like in [11], we place the poles in a circle of center $\sigma$ and radius $r$ by replacing $A_{a_{i}}-B_{a_{i}} K_{i}$ by $\frac{A_{a_{i}}-B_{a_{i}} K_{i}-\sigma I}{r}$. This particular region is depicted in Figure 6. With this change and taking $P_{i}^{-1}=Q_{i}, P_{j}^{-1}=Q_{j}$ and $U_{i}=K_{i} P_{i}^{-1}$ (26) is obtained.

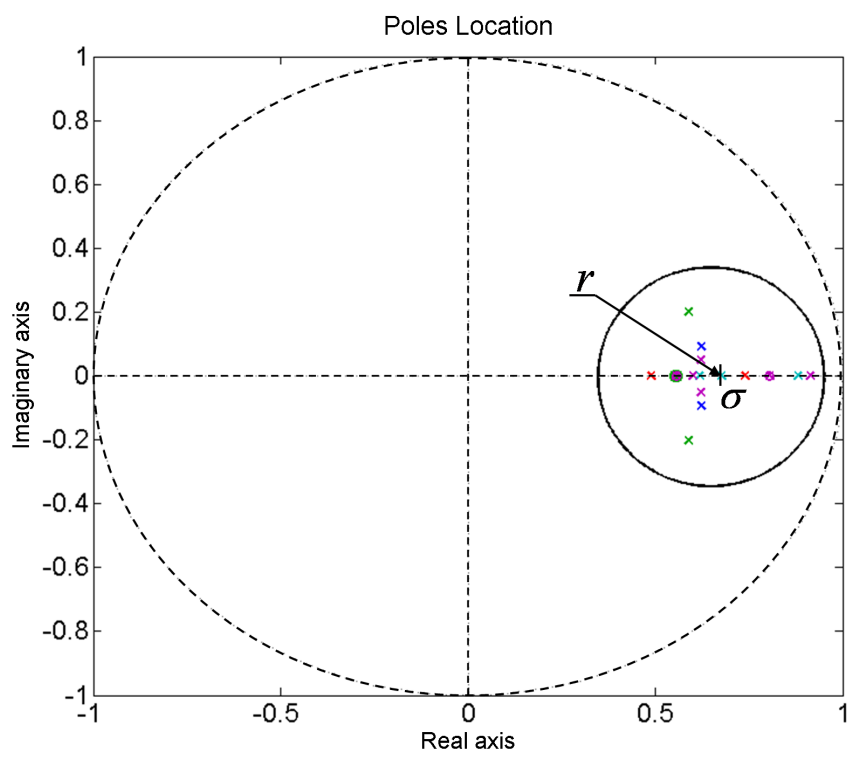

Fig. 6. Pole placement region.

\section{Simulation Results}

For simulation, we take the on-ramp neighborhood depicted in Figure 5. We choose the following parameters (cells are homogenous):

- Cell length $l=0.3 \mathrm{~km}$

- Free flow speed $v=80 \mathrm{~km} / \mathrm{h} \pm 5 \%$

- Backward congestion speed $v=35 \mathrm{~km} / \mathrm{h} \pm 15 \%$

- Maximum flow $\phi_{M}=7000 \mathrm{~km} / \mathrm{h} \pm 8 \%$

These parameters values and uncertainties ranges are chosen according to experimental data.

We take the following weighting constants: $f_{d}=1, f_{z}=$ $1 / 200, f_{J}=1 / 22, f_{p}=1, f_{w}=1 / 4$. These constants are chosen with respect to the range of disturbance values.

To compute our controllers, we take $\sigma=0.6$ and $r=0.35$ for transition $\tau_{i j}, i=j$ and $\sigma=0$ and $r=1$ for transition $\tau_{i j}, i \neq j$, this means that we doesn't place pole $<$ for the switching between modes. This leads to reduce the conservatism of our approach. The result of pole placement for each of the 6 cases is depicted in Figure 6.

We detail our results for case 4 . We obtain the following 
state feedback gain:

$$
K_{4}=\left(\begin{array}{lllll}
221.24 & 216.49 & 191.24 & 113.05 & 36.36
\end{array}\right.
$$

The sensitivity functions between disturbances and $\rho_{3}$ are given in Figure 7, we can see only 6 sensitivity function because the matrix $E_{a_{4}}$ has several columns of zeros. The attenuation is good in low frequency, where disturbances occurs [10].

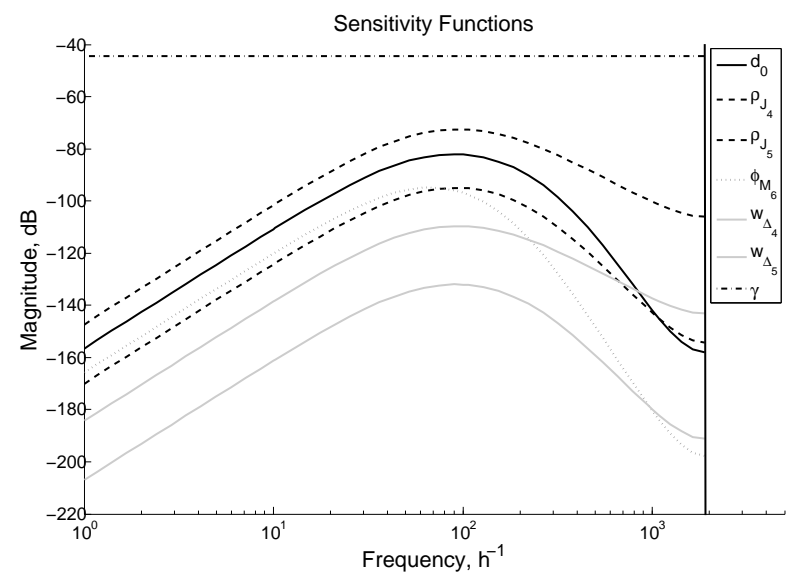

Fig. 7. Sensitivity functions.

In the scenario we have chosen, the system has to track a congestion front which propagates backward then forward. The system goes from case 1 to case 5 via all the intermediate cases then goes back to case 1 . The result of the simulation is depicted in Figure 8. We can see that the system is following the congestion front despite parametric uncertainties and disturbance. The active controller $\left(K_{1}\right.$ to $\left.K_{5}\right)$ is chosen with respect to traffic condition. The choice of this controller is based on the nominal parameters of the system and so the controller chosen may not be the appropriate one. This can explain the switching problem that occurs around $t=1.7 \mathrm{~h}$ when the system switches from case 3 to case 2 .

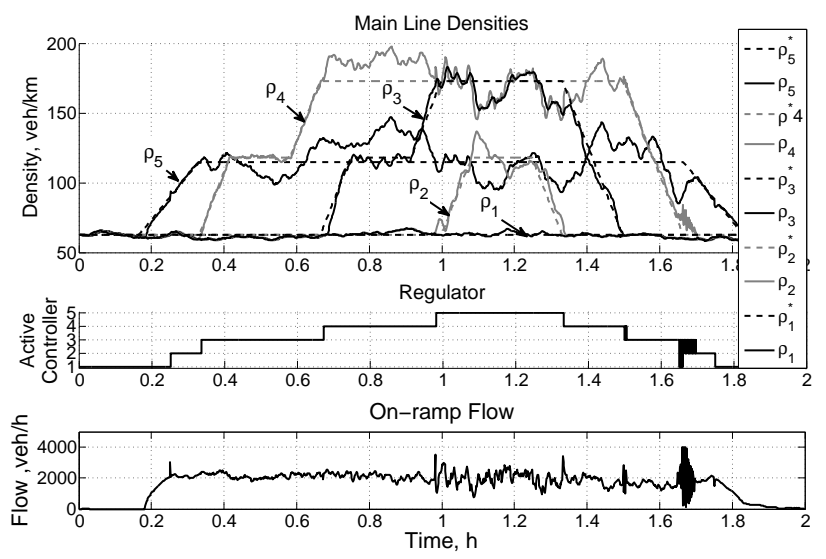

Fig. 8. Simulation results.

\section{Conclusions}

In this paper, the CTM has been extended with parametric uncertainties. A new robust switched $H_{\infty}$ PI controller has been designed, taking account of the uncertainty model. The simulation result shows that this solution is efficient to track congestion front, and shows a good attenuation of disturbance. Some improvements on switching conditions and queue regulation still have to be done.

\section{REFERENCES}

[1] C. F. Daganzo, "The cell-transmission model, part 2: Network traffic," Transportation Research Part B, vol. 29, pp. 79-94, 1995.

[2] C. F. Daganzo, "The cell-transmission model, a dynamic representation of highway traffic consistent with the hydrodynamic theory," Transportation Research Part B, vol. 28, pp. 269-288, 1994.

[3] N. Geroliminis and C. F. Daganzo, "Existence of urban-scale macroscopic fundamental diagram : Some experimental findings," Transportation Research Part B, no. 42, pp. 759-770, 2008.

[4] G. Gomes and R. Horowitz, "Optimal freeway ramp metering using the asymetric cell transmition model," Transportation Research Part $C$, vol. 14, pp. 244-262, 2006.

[5] H. Haj-Salem, P. Poirier, J.-F. Heylliard, and J.-P. Peynaud, "Alinea: a local traffic responsive strategy for ramp metering: Field results on a6 motorway in paris," in IEEE Intelligent Transportation Systems Conference Proceedings, Oakland, USA, August 2001, pp. 106-111.

[6] D. Jacquet, "Freeway traffic management using linear programming," Proceedings of the 17th IFAC World Congress, 2008.

[7] D. Jacquet, J. Jaglin, D. Koenig, and C. C. D. Wit, "Non-local feedback ramp metering controller design," Proceedings of the 11th IFAC Symposium on Control in Transportation Systems, 2008.

[8] D. Jacquet, "Modelisation macroscopique du trafic et controle des lois de conservation non lineaires associees," Ph.D. dissertation, INP Grenoble, LAG, 2007.

[9] A. Kotsialos and M. Papageorgiou, "A hierarchical ramp metering control scheme for freeway networks," in Proceedings of the American Control Conference, Portland, June 2005, pp. 2257-2262.

[10] A. Lemarchand, J. J. Martinez, and D. Koenig, "Hierarchical coordinated freeway on-ramp metering using switching system theory," in IFAC Symposium on Structured System Control, Ancona, Italy, September 2010.

[11] V. Montagner, V. Leite, and P. Peres, "Discrete-time switched systems : Pole location and structural constrained control," in Proceedings of the Conference on Decision and Control, Maui, Hawaii USA, December 2003, pp. 6442-6447.

[12] L. Munoz, X. Sun, D. Sun, G. Gomes, and R. Horowitz, "Methodological calibration of the cell transmission model," in Proceedings of the American Control Conference, Boston massachusetts, july 2004, pp. $798-803$.

[13] D. Schrank and T. Lomax, "Mobility report," 2007.

[14] Y. J. Stephanedes and K.-K. Chang, "Optimal control of freeway corridors," ASCE Journal of Transportation Engineering, vol. 119, pp. 504-514, 1993.

[15] X. Sun and R. Horowitz, "A localized switching ramp-metering controller with a queue lenght regulator for congested freeways," in Proceedings of the American Control Conference, june 2005.

[16] _ _ "Set of new traffic-responsive ramp-metering algorithms and microscopic simulation results," Journal of the Transportation Research Board, vol. 1959, pp. 9-18, 2006. 